\title{
Microrna -145 Expression Inhibits Invasion and Metastasis of Cervical Cancer Cells
}

\author{
Shun-qing ZHOU ${ }^{1}$, Ning $\operatorname{LIU}^{1}$ and Yang $\operatorname{LIN}^{1, a, *}$ \\ ${ }^{1}$ Department of Obstetrics and Gynecology, The Second Hospital, Jilin University, \\ \#218 Ziqiagn Street, Nanguan District, Changchun 130041, China \\ alinyang1155@163.com \\ ${ }^{*}$ Corresponding author
}

Keywords: Cervical Cancer, Microrna-145, Invasion, Metastasis.

\begin{abstract}
Purposes] To investigate the expression of microRNA-145 on invasion and metastasis of cervical cancer cells. [Methods] Analysis of the relationship between high expression of microRNA-145 on invasion and metastasis of cervical cancer cells by Transwell invasion assay and Wound scratch assay. [Results] There is a certain correlation between inhibit the invasion and metastasis and microRNA-145 high expression in cervical cancer cells $(\mathrm{P}<0.05)$. [Conclusions] MicroRNA-145 inhibiting cervical cancer invasion and metastasis in the occurrence and development of cervical cancer. Can be used for targeted therapy of cervical cancer and prognosis judgement index.
\end{abstract}

\section{Introduction}

Cervical cancer is one of the most common gynecological malignancies, in recent decades, due to the universal application of cervical cytology screening, cervical cancer to early detection and treatment, reducing the mortality of cervical cancer. But in recent years because of its incidence of a younger trend, and morbidity and mortality is still high, a serious threat to the majority of women's physical and mental health.

MicroRNA-145 is a kind of eukaryotic endogenous regulatory function of non-coding RNA, the size of about 20 to 25 nucleotides. And to instruct the silencing complex to degrade target mRNA or repress target mRNA translation. It has been shown that miRNAs are involved in the regulation of malignant tumorigenesis and the proliferation, invasion and migration of tumor cells. MicroRNA-145 (miR-145), an important member of miRNA, has been shown to be down-regulated in many tumor tissues, such as cervical cancer, ovarian cancer, and endometrial cancer ${ }^{[1-3]}$. It is suggested that miR-145 may act as a tumor suppressor gene and regulate tumorigenesis and development ${ }^{[4]}$. To investigate the role of miR-145 in the development of cervical cancer, we investigated the effect of microRNA-145 expression on invasion and migration of cervical cancer cells by tumor cell invasion assay and tumor migration assay. And to provide theoretical basis for treatment.

\section{Materials and Methods}

\section{Study Subjects}

Hela cells were divided into 2 groups: (1): experimental group (M) transfected with microRNA-145; (2) control group (NC) transfected with miR-145 mimetic negative control. 


\section{Reagents and Methods}

\section{Reagents}

Cell culture medium, fetal bovine serum, diluted liposome transfection medium and cell digestion solution, Lipofectamine 2000 transfection reagent, miR-145 mimics and miR-145 mimics negative control Pre-miR-145 mimics, BD matrigel, Matrigel, Transwell, polycarbonate membrane, $8 \mu \mathrm{m}$ pore size, $0.1 \%$ cell stained crystal violet solution.

\section{Methods}

Tumor cell invasion test: the two groups of cells were added to the DMEM medium containing $0.1 \%$ FBS for 18 hours, then the two groups of cells in the transwell chamber indoors, polycarbonate membrane permeability, the indoor cells can And the influence of the components of the lower culture on the growth and movement of the cells was examined by the influence of the components in the lower culture. Wipe off the matrix with a cotton swab and the cells in the room after the crystal violet staining. Randomly selected field of view, observation and photographs, calculated through the polycarbonate membrane cell number.

Tumor migration experiments: In the experimental group and the control group, the central area of the cell growth with a trace of the head crossed, the central part of the cells were removed, and then added serum-free medium, and then placed in 37 degrees $5 \% \mathrm{CO} 2$ incubator, continue to cultivate. $(0,6,12,24$ hours $)$, remove the cell culture plate to observe whether the central scars area of peripheral cell growth, in order to determine the cell migration ability, according to 0,6,12,24 hours Sampling, taking pictures.

\section{Statistical Methods}

SPSS V.19 statistical software was used to analyze the experimental data. The data were expressed as mean \pm standard deviation $(X \pm S)$. The independent samples $t$ test was used to compare the two groups, $\mathrm{P}<0.05$

\section{Results}

\section{Comparison of Invasive Ability of Cells}

We used the miR-145 mimetic transfected cells and the miR-145 mock negative control cells to carry out the invasion experiment. Results is shown in Fig. 1 and Table 1.The significant difference between the experimental group and the control group was 0.029 , $\mathrm{P}<0.05$, demonstrating that over expression of miR-145 significantly inhibited the invasion ability of the cells.

Table 1. expression of miR-145 in tissues from the experimental group and the control group

\begin{tabular}{|c|c|c|}
\hline Group & Number of cases & Number of transmural cells (a) \\
\hline Control group & 6 & $42.83 \pm 8.5$ \\
\hline test group & 6 & $31.67 \pm 6.53$ \\
\hline
\end{tabular}




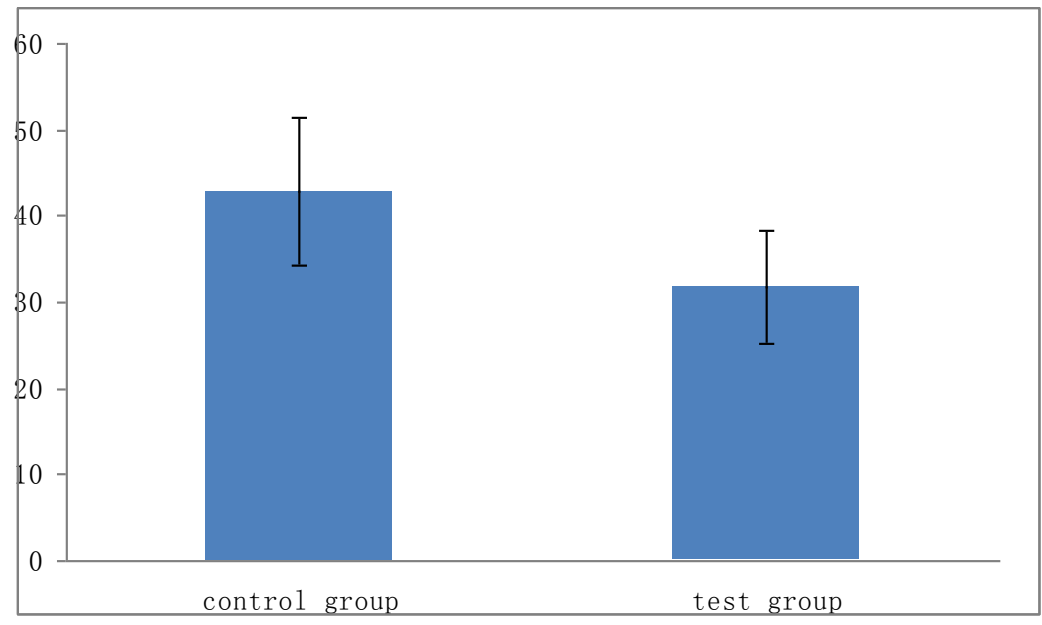

Fig.1. Comparison of cell invasiveness of cells transfected with miR-145 mimics and miR-145 mimics negative control

\section{Comparison of Cell Migration Ability}

We performed migration assays using cells transfected with miR-145 mimics and cells with miR-145 mock negative controls, respectively. Scratch experiments showed that the migration ability of cells transfected with miR-145 mimics was significantly different over the long-term levels compared to the control group. The results confirmed that over expression of miR-145 significantly inhibited the migration of tumor cells. As shown in Fig. 2 and Table 2.

Table 2. Comparison of cell migration ability of cells transfected with miR-145 mimics and miR-145 mimics negative control

\begin{tabular}{|c|c|c|c|c|c|c|c|}
\hline Group & cases & 0 hours & 6 hours & 12 hours & 24 hours & $\mathrm{F}$ & $\mathrm{P}$ \\
\hline $\begin{array}{l}\text { Control } \\
\text { group } \\
\text { (Scratch } \\
\text { spacing } \\
\text { (Pixels)) }\end{array}$ & 15 & $\begin{array}{r}714.20 \\
\pm 38.17\end{array}$ & $\begin{array}{c}338.40 \\
\pm 35.22 * *\end{array}$ & $\begin{array}{c}291.67 \\
\pm 24.78 * *\end{array}$ & $\begin{array}{c}228.80 \\
\pm 28.65 * *\end{array}$ & 693.952 & $\begin{array}{c}<0.00 \\
1\end{array}$ \\
\hline $\begin{array}{l}\text { test group } \\
\text { (Scratch } \\
\text { spacing } \\
\text { (Pixels) })\end{array}$ & 15 & $\begin{array}{r}680.67 \\
\pm 51.72\end{array}$ & $\begin{array}{c}371.60 \\
\pm 31.37 * *\end{array}$ & $\begin{array}{c}314.47 \\
\pm 46.66 * *\end{array}$ & $\begin{array}{c}299.73 \\
\pm 51.66 * *\end{array}$ & 225.391 & $\begin{array}{c}<0.00 \\
1\end{array}$ \\
\hline $\mathrm{t}$ & 2.02 & 3.02 & 4.02 & 5.02 & & & \\
\hline $\mathrm{p}$ & 2.02 & 3.02 & 4.02 & 5.02 & & & \\
\hline \multicolumn{8}{|c|}{$* * \mathrm{P}<0.01$} \\
\hline
\end{tabular}




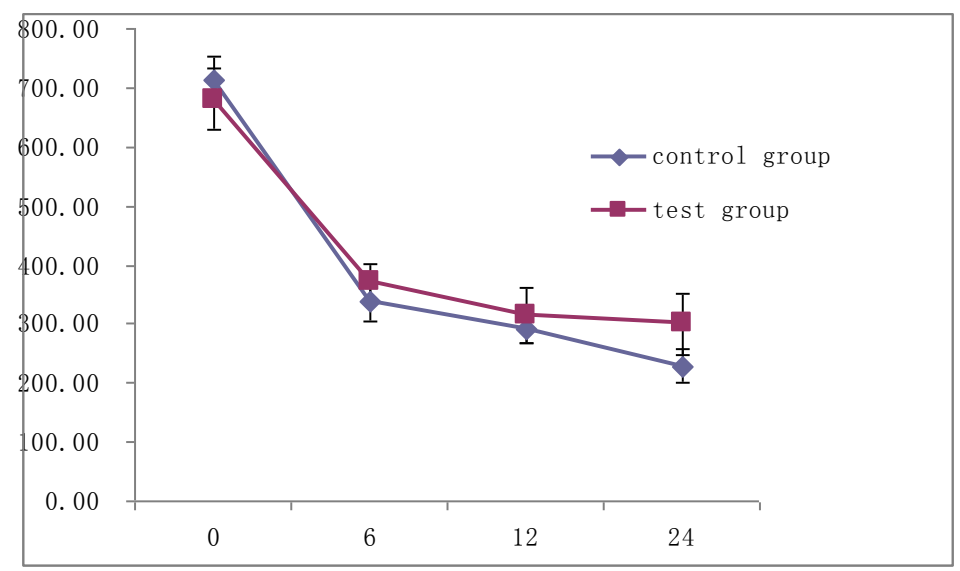

Fig.2. Comparison of cell migration ability of cells transfected with miR-145 mimics and miR-145 mimics negative control

\section{Discussion}

Cervical cancer because of its high incidence, younger trends and how early diagnosis has been the concern of researchers, the current treatment of cervical cancer with surgery and radiotherapy-based, supplemented by an integrated program of chemotherapy. Sexual dysfunction after cervical cancer treatment is the most persistent factor affecting the long-term quality of life of patients with cervical cancer. So gene therapy has become another hot spot.

At present, the development of cervical cancer, especially the genes involved in the development of cervical cancer and its mechanism play a research hotspot.

The expression of miR-145 in cancer cells was significantly reduced. Experiments by directing specific target mRNA allows us to more effectively investigate the major functions and molecular mechanisms of miR-145. Our further study of miR-145 in the treatment of cervical cancer may provide a valuable role in the response. The results showed that over expression of miR-145 could inhibit the viability of cervical cancer in vitro and in vivo, and induce apoptosis.

MiR-145 is an endogenous non-coding small molecule RNA involved in the regulation of cell proliferation, invasion and migration. This function is related to the effect of miR-145 on p53 gene expression. Activation of p53 induces miR-145 subsequent silencing of c-Myc and its downstream genes, miR-17 and elF4E, to influence tumorigenesis, cell proliferation and protein synthesis. Although p53 has been shown to inhibit the transcription of the c-Myc gene[5,6], miR-145 also plays a more important role in this respect because anti-MIR-145 blocks the vast majority of p53-mediated genes inhibition. C-Myc gene can enhance the expression of p53 ${ }^{[7]}$. In this regulatory pathway miR-145 is another important role is to improve the activity of p53, the total known, p53 is a tumor suppressor gene, DNA damage can activate p53 and further induce the cell cycle, to stagnate, can also induce cells Of apoptosis. P53-induced miR-145 expression, in addition, wild-type p53 on miR-145 expression in cervical cancer cells is essential. Furthermore, we found that radiation did not induce the effect of miR - 145 expression in C - 33A cells in the port mutant p53. In addition, p53 can induce the expression of p21 by direct or indirect action. Therefore, this is a complex regulatory system, the interruption of this balance system, such as miR-145 expression down, may lead to tumor cell invasion and metastasis [8].

Micro RNAs are often released, and they may serve as potential tumor biomarkers. In 
particular, down regulation of miR-145 seems to be associated with precancerous lesions ${ }^{[9]}$, and it has been shown to be a biomarker for early detection of cancer. Recent studies have shown that tumor-derived micro RNAs can be detected in blood or serum ${ }^{[10]}$, thus opening a new biomarker discovery pathway. Therefore, miR-145 can be used as a novel biomarker because it can be easily detected in real-time quantitative RT-PCR [11].

Abnormal expression of miRNAs in cervical cancer. Most miRNAs are located in the fragile genes associated with tumor formation, suggesting that miRNAs play an important role in tumorigenesis. Therefore, it can be used as a new tumor suppressor gene or oncogene, miR-145 in cervical cancer expression levels of up-regulation of tumor suppressor gene activity. In this study, miR-145 was transfected into the cell model of miR-145 mimics and negative control cells, and the invasion of cervical cancer cells and migration of cervical cancer cells were studied. The invasion and migration of cervical cancer cells were confirmed by the expression of miR-145. The results showed that the expression of miR-145 in cervical cancer cells, the invasion of tumor cells $(\mathrm{P}<0.01)$ and the migration of tumor cells $(\mathrm{P}<0.05)$ can be inhibited significantly. MiR-145 is closely related to invasion and metastasis of cervical cancer cells, which are closely related to tumor development and poor biological behavior, suggesting that low expression of miR-145 in cervical cancer may indicate poor prognosis .

Induction of miR-145 expression up regulates miR-145, which ultimately contributes to radiosensitization in cervical cancer cells. ${ }^{[12]}$ Of course, we need to further study it to exploit more specific miR-145 radiosensitization mechanisms. Some studies have shown that miR-145 contributes to radiosensitization. These findings demonstrate that miR-145 may be an important biomarker for prognostic evaluation and a novel radiation-sensitive target in cervical cancer, which may help to establish new therapeutic strategies and improve the therapeutic efficacy of cervical cancer.

Therefore, miR-145 can be used as a new biological marker in the diagnosis of cervical cancer by using miR-145 as a targeted therapy for cervical cancer, which will provide a new therapeutic approach for cervical cancer. s method. At present stage of gynecological malignancies in the treatment of traumatic long-term prognosis and difficult to monitor the shortcomings of miR-145 research and application of gynecological malignancies will be the diagnosis and treatment of new biological markers and targeted therapy new method.

\section{Acknowledgement}

This research was financially supported by Norman Bethune Program of Jilin University [2015213].

\section{References}

[1] Cabrita MA, Bose R, Vanzyl EJ, Pastic A, Marcellus KA, Pan E, Hamill JD, McKay BC. The p53 protein induces stable miRNAs that have the potential to modify subsequent p53 responses.Gene. 2017. S0378-1119(17)30039-2.

[2] Huang L, Lin J X, Yu Y H, et al. Down regulation of six micro RNAs is associated with advanced stage, lymph node metastasis and poor prognosis in small cell carci -noma of the cervix[J]. PLoS One,2012,7(3):E33762. 
[3] Wu W, Lin Z, Zhuang Z, et al. Expression profile of mammalian micro RNAs in endometrioid adenocarcinoma [J]. Eur J Cancer Prev, 2009, 18(1):50-55.

[4] Sachdeva M,Mo Y Y.miR-145-mediated suppression of cell growth, invasion and metastasis [J]. Am J Transl Res, 2010, 2(2):170-180.

[5] Mataki H, Seki N, Mizuno K, Nohata N, Kamikawaji K, Kumamoto T, Koshizuka K, Goto Y, Inoue H. Dual-strand tumor-suppressor microRNA-145 (miR-145-5p and miR-145-3p) coordinately targeted MTDH in lung squamous cell carcinoma. Oncotarget. 2016,7(44):72084-72098.

[6] Zhou X, Yue Y, Wang R, Gong B, Duan Z. MicroRNA-145 inhibits tumorigenesis and invasion of cervical cancer stem cells. Int J Oncol. doi: 10.3892/ijo.2017.3857.

[7] Sachdeva M, and Mo YY. p53 and c-myc: howdoes the cell balance "yin" and "yang"? Cell Cycle.2009,8:1303.

[8] Han Q, Zhang HY, Zhong BL, Wang XJ, Zhang B, Chen H. MicroRNA145 Inhibits Cell Migration and Invasion and Regulates Epithelial- Mesenchymal Transition (EMT) by Targeting Connective Tissue Growth Factor (CTGF) in Esophageal Squamous Cell Carcinoma.Med Sci Monit. 2016, 22:3925-3934.

[9] Huang Z, Huang D, Ni S, Peng Z, Sheng W and Du X. Plasma microRNAs are promising novel biomarkers for early detection of colorectal cancer. Int J Cancer 2009.

[10] Han J, Sun Y, Song W, Xu T.Dev Comp Immunol. 2017,68:79-86. doi: 10.1016/ j.dci.2016.11.021.

[11] Liu HT, Xu YT, Li HY, Zhao J, Zhai HY, Chen Y. Loss of microRNA- 145 expression is involved in the development and prognosis of breast cancer complicated by type 2 diabetes mellitus.Int J Biol Markers. 2016,31(4):e368-e374.

[12] Zhu W, Qin W, Atasoy U and Sauter ER. Circulating micro RNAs in breast cancer and healthy subjects. BMC Res Notes 2009, 2: 89. 